\title{
STUDIES OF RADIO SOURCES AT I59 MC/S
}

\author{
S. ARCHER, J. E. BALDWIN, D. O. EDGE, B. ELSMORE, P. A. G. SCHEUER,
}

\author{
AND J. R. SHAKESHAFT \\ Mullard Radio Astronomy Observatory, Cavendish Laboratory \\ Cambridge, England
}

Two years ago the Cambridge 4-aerial interferometer [1] was adapted to work at a frequency of $159 \mathrm{Mc} / \mathrm{s}$, the resolving power thereby being increased by a factor of four over that at the previous frequency, $81.5 \mathrm{Mc} / \mathrm{s}$. The overall beamwidth at $159 \mathrm{Mc} / \mathrm{s}$ is 1.2 degrees by 7 degrees but the beam contains interference fringes in two planes at right angles, so that, by phase-switching between the east pair and the west pair of aerials, sources with angular diameters greater than about 7 minutes of arc are eliminated. A survey using this technique has been carried out by Edge and Shakeshaft and may be called a "small diameter" survey. If the phase-switching receiver is connected between the north pair and the south pair of aerials it is possible to record sources with diameters up to about 1 degree and to measure diameters between 2 and 8 minutes of arc. Archer and Baldwin have used the aerial in this way to make a "large diameter" survey. These two surveys will be referred to collectively as the $3 \mathrm{C}$ survey.

The reduction of the $3 \mathrm{C}$ small diameter records has been completed exhaustively for two regions: $00^{\mathrm{h}}<\alpha<24^{\mathrm{h}},+37^{\circ}<\delta<+52^{\circ}$ and $00^{\mathrm{h}}<\alpha<08^{\mathrm{h}}$, $-10^{\circ}<\delta<+10^{\circ}$, the latter region being chosen to overlap the survey of Mills and Slee (MS) [2], in view of the large discrepancies between their survey with the 50 -minute-of-arc pencil beam of the Mills cross and the earlier Cambridge survey [3]. In addition, preliminary positions have been derived for the remainder of the sky between declinations -10 and +57 degrees.

Factors affecting the detection of sources include the sensitivity and "confusion" due to several sources contributing to the deflection at any instant. The former is unimportant in the $2 \mathrm{C}$ and $3 \mathrm{C}$ surveys, the noise being negligible, but it sets the limit to the MS survey. The $2 \mathrm{C}$ and $3 \mathrm{C}$ surveys are limited by confusion, though it is worth while noting that this effect is not peculiar to interferometers, but is related in any system to the solid angle over which sources may contribute to the output.

Also to be considered are the effects of angular diameters of sources and possible side lobes of the reception pattern. The Mills cross detects all sources that produce a sufficient flux density within the reception pattern, so that the limiting flux density is constant for all sources smaller than $50 \times 50$ minutes of arc and increases for sources with larger angular diameters. With the interferometer, as already pointed out, there is a systematic bias 
against large diameter sources, and since in any class these will generally be the brightest there is a bias against intense sources; therefore any apparent deficit must be corrected for this selection effect.

Because of the large range in brightness of radio sources, care must be taken to ensure that the passage of intense sources through subsidiary maxima of the reception pattern does not produce responses that are listed as true sources. The danger is particularly great for aerials with "incompletely filled" apertures.

A comparison of the positions of $2 \mathrm{C}$ and $3 \mathrm{C}$ sources shows poor agreement, and although in the $2 \mathrm{C}$ catalog the majority of sources with flux densities less than $25 \times 10^{-26}$ watts $\mathrm{m}^{-2}(\mathrm{c} / \mathrm{s})^{-1}$, i.e., about one-third the total number, were indicated as unreliable, this was evidently an optimistic estimate and $40 \times 10^{-26}$ watts $\mathrm{m}^{-2}(\mathrm{c} / \mathrm{s})^{-1}$ would be a more realistic figure. This value is confirmed by consideration of the statistical properties of the record. Since four times as many sources can be distinguished to the same level of confidence in the $3 \mathrm{C}$ survey as in the $2 \mathrm{C}$ survey, it is thought that about 100 sources per steradian should be reliable in the $3 \mathrm{C}$ survey, i.e. those with flux densities greater than $7.5 \times 10^{-26}$ watts $\mathrm{m}^{-2}(\mathrm{c} / \mathrm{s})^{-1}$ at $159 \mathrm{Mc} / \mathrm{s}$.

A search has been made for MS sources in the $3 \mathrm{C}$ list and, if absent there, on the original $3 \mathrm{C}$ records. Out of 207 sources 67 have been found, the expected number of random coincidences being 32 . The proportion of course is greater for the more intense, and only 4 out of the top 28 sources are not accounted for. That still leaves 140 sources. In the case of 63 of these, the $3 \mathrm{C}$ evidence is inconclusive, but 77 sources definitely do not appear on the $3 \mathrm{C}$ small-diameter records. This may mean that they have $(a)$ angular diameters greater than 5 minutes of arc or $(b)$ peculiar spectra, or that they are $(c)$ spurious side-lobe responses. We shall see later that such a high proportion (77 out of 207) of extended sources is unlikely.

The search has also been done the other way round to see how many of the $3 \mathrm{C}$ sources can be explained in terms of MS sources. Of 162, 66 are confirmed directly, a further 37 may originate in the same physical objects as nearby sources in the MS list, and 59 have no corresponding sources but they have flux densities near the lower limit of the MS survey.

The MS sources listed as extended have been looked for on the $3 \mathrm{C}$ large diameter records. Out of 18 , no trace could be found of 8 .

There is fair agreement about sources with flux densities more than $20 \times 10^{-26}$ watts $\mathrm{m}^{-2}(\mathrm{c} / \mathrm{s})^{-1}$ at $85 \mathrm{Mc} / \mathrm{s}$, i.e., some hundreds over the whole sky, but it is an understatement to say that the general position is unsatisfactory and that independent checks are required.

In an attempt to make such a check we have had the new array at Cambridge, the collecting area of which is 450 by $18 \mathrm{~m}$, working with a total power receiver at a frequency of $178 \mathrm{Mc} / \mathrm{s}$. The beam is 18 minutes of arc in right ascension by 5 degrees in declination. There has been time for only one week's observations since the completion of the array and this is clearly inadequate, but some tentative conclusions have been reached. For the area $00^{\mathrm{h}}<\alpha<08^{\mathrm{h}},-10^{\circ}<\delta<+10^{\circ}$ all sources in the MS survey hav- 
ing fluxes greater than $40 \times 10^{-26}$ watts $\mathrm{m}^{-2}(\mathrm{c} / \mathrm{s})^{-1}$ at $85 \mathrm{Mc} / \mathrm{s}$ have been compared with the total-power records as have those in the $3 \mathrm{C}$ survey with fluxes greater than $15 \times 10^{-26}$ watts $\mathrm{m}^{-2}(\mathrm{c} / \mathrm{s})^{-1}$ at $159 \mathrm{Mc} / \mathrm{s}$. Both surveys should be reliable above these levels. In four cases sources from the lists with fluxes less than these values have been included since they are plainly the same objects as sources in the other already included. The conclusions are:

(a) All of the $163 \mathrm{C}$ sources are confirmed.

(b) In 14 cases there is excellent agreement between all three surveys.

(c) One source in the $3 \mathrm{C}$, but not the MS list, is confirmed.

(d) One MS source, resolved into two $3 \mathrm{C}$ sources, is seen to be two sources.

(e) Two MS sources, which are not in the $3 \mathrm{C}$ list, cannot be confirmed or otherwise as the evidence is inadequate.

(f) Of three of the MS sources, no trace can be found.

I would emphasize again that these are only preliminary results and much more work is needed on this problem.

In order to check some of the positions Elsmore [4] has used the aerial as a crossed-axis interferometer to observe 17 intense sources. He describes this work in a paper presented to the Symposium and his list shows good agreement with the $3 \mathrm{C}$ positions.

The problem of identifications has been in the hands of Dr. Dewhirst, who will be telling us of his results. Baldwin and Edge [5] have made special efforts to determine the positions of the intense sources near the remnants of the supernovae of $\mathrm{AD} 1572$ and 1604. In the former case a discrepancy between the radio position and that of Tycho Brahe has been shown by Minkowski to be due to an error in Tycho's position.

Statistical studies have been carried out for the limited regions described. These are intended to reveal information on the distribution of sources in space and are of two kinds: Firstly, counts of individual sources $N(S)$ down to various levels $S$ of flux density should follow the relationship $N(S) \propto S^{-3 / 2}$ if sources are distributed uniformly through space. The analysis of the $2 \mathrm{C}$ survey showed an excess of faint sources (or a deficit of intense ones) [6], but confusion effects [7] may have been more serious than was supposed. The $3 \mathrm{C}$ results (for regions away from the galactic plane) show a similar effect though less marked. For 85 sources in the strip from $+37^{\circ}<\delta<+52^{\circ}$ the slope of the graph of $\log N$ against $\log S$ is -2.2 . For the strip $-10^{\circ}<\delta<10^{\circ}$, the slope is -2.7 for the $3 \mathrm{C}$ results as compared with -2.5 for the $2 \mathrm{C}$ sources. Confusion effects should be much larger for the $2 \mathrm{C}$ results, so that the agreement here would seem to indicate that the systematic effects of confusion have not seriously affected them. There is a possibility, however, that the decreased confusion at $159 \mathrm{Mc} / \mathrm{s}$ may be compensated by an increased effect of the angular diameters of sources.

Because of the uncertainty about confusion and the subjective difficulties in abstracting sources from records Scheuer [8] has computed the expected statistical properties of the record deflections on the assumption of a uniform distribution and has compared it with the observed distribution. Such a 
procedure completely eliminates any confusion errors but is still subject to errors due to finite angular diameters.

The results are given in Tables I and II, in which are plotted the number of deflections in different ranges of intensity, at $159 \mathrm{Mc} / \mathrm{s}$ and $81.5 \mathrm{Mc} / \mathrm{s}$, respectively. The observed and computed numbers have been normalized by equalizing the number of deflections in the lowest range. Table I shows good agreement between the two sets to $\sigma=8.65$ but for larger values of $\sigma$ the observed numbers are consistently less, except in the highest range where nearly all the deflections are caused by Virgo A. A similar effect occurs in Table II but it sets in sooner, at $\sigma=3.15$. It can be shown [9] that this difference is to be expected if the discrepancy is caused by a true deficit of intense sources.

\begin{tabular}{|c|c|c|c|c|c|}
\hline \multirow{3}{*}{$\begin{array}{l}0^{\sigma} \\
0.869\end{array}$} & \multicolumn{2}{|l|}{$\begin{array}{l}\text { TABLE I } \\
(159 \mathrm{Mc} / \mathrm{s})\end{array}$} & \multicolumn{3}{|c|}{$\begin{array}{l}\text { TABLE II } \\
(81.5 \mathrm{Mc} / \mathrm{s})\end{array}$} \\
\hline & $\begin{array}{l}\text { Number } \\
\text { observed }\end{array}$ & $\begin{array}{l}\text { Number } \\
\text { computed }\end{array}$ & $0^{\sigma}$ & $\begin{array}{l}\text { Number } \\
\text { observed }\end{array}$ & $\begin{array}{l}\text { Number } \\
\text { computed }\end{array}$ \\
\hline & 1056 & 1056 & 0 & 2443 & 2443 \\
\hline & 750 & 749.5 & 0.79 & 2040 & 1816 \\
\hline 2.191 & 153 & 137.8 & 2.36 & 419 & 497 \\
\hline $\begin{array}{l}4.300 \\
6.505\end{array}$ & 29 & 29.2 & & 131 & 175 \\
\hline $\begin{array}{l}6.505 \\
8.65\end{array}$ & 8 & 12.3 & 3.15 & 46 & 85 \\
\hline $\begin{array}{r}8.00 \\
10.64\end{array}$ & 2 & 5.9 & $\begin{array}{l}3.93 \\
4.72\end{array}$ & 35 & 48 \\
\hline 12.67 & 1 & 3.75 & 5.51 & 14 & 30 \\
\hline 14.61 & 1 & 2.3 & 6.29 & 7 & 21 \\
\hline 20.45 & 0 & 3.9 & 7.08 & 13 & 16 \\
\hline & & & 7.87 & 4 & 12 \\
\hline$>20.45$ & 6 & 5.4 & $>7.87$ & 57 & 64 \\
\hline$>8.65$ & 10 & 21.2 & $>3.15$ & 176 & 277 \\
\hline $\begin{array}{c}8.65 \\
\text { to } \\
20.45\end{array}$ & 4 & 15.85 & $\begin{array}{c}3.15 \\
\text { to } \\
7.87\end{array}$ & 119 & 213 \\
\hline
\end{tabular}

The effects of angular diameters on these figures must be examined. Data on source diameters away from the plane are scanty, but Baldwin and Archer have determined upper limits to the diameters of the 27 brightest sources in the region $-10^{\circ}<\delta<+47^{\circ},|b|>20^{\circ}$ and find that 25 have diameters $>5^{\prime}$, $21>3.5$, and $10>2.5$. (These results seem to agree with those obtained at Nançay but are difficult to reconcile with the large proportion of extended 
sources reported by Mills in the previous paper.) (If a distribution of sources with angular diameters equivalent to these quoted upper limits is assumed it is just possible to account for the deficit in Table I but not for that in Table II. An independent argument [9] shows that the two tables taken together are incompatible with a uniform distribution of sources whatever the angular diameters or clustering tendencies of the sources may be. This apparent deficit of intense sources chiefly concerns those with flux densities greater than $30 \times 10^{-26}$ watts $\mathrm{m}^{-2}(\mathrm{c} / \mathrm{s})^{-1}$ at $159 \mathrm{Mc} / \mathrm{s}$. If the deficit is isotropic there are only about 100 sources brighter than this; whereas, on the basis of a uniform distribution and the observed numbers of fainter sources, some 200 would be expected.

\section{REFERENCES}

[1] Ryle, M., and Hewish, A. Mem. R.A.S. 67, 97, 1955.

[2] Mills, B. Y., and Slee, O. B. Aust. J. Phys. 10, 162, 1957.

[3] Shakeshaft, J. R., Ryle, M., Baldwin, J. E., Elsmore, B., and Thomson, J. H. Mem. R.A.S., 67, 106, 1955.

[4] Elsmore, B. (In press.)

[5] Baldwin, J. E., and Edge, D. O. The Observatory, 77, 139, 1957.

[6] Ryle, M., and Scheuer, P. A. G. Proc. Roy. Soc. A. 230, 448, 1955.

[7] Bolton, J. G. The Observatory, 76, 62, 1956.

[8] Scheuer, P. A. G. Proc. Camb. Phil. Soc. 53, 764, 1957.

[9] Edge, D. O., Scheuer, P. A. G., and Shakeshaft, J.R. M.N.R.A.S. 118, 183, 1958.

\section{Discussion}

Mills: What were the actual numbers of independent deflections used in this analysis?

Shakeshaft: In determining the probability distribution of record deflections at $159 \mathrm{Mc} / \mathrm{s}$ the deflections are independent, having been measured once per beamwidth (measured between zeros). 